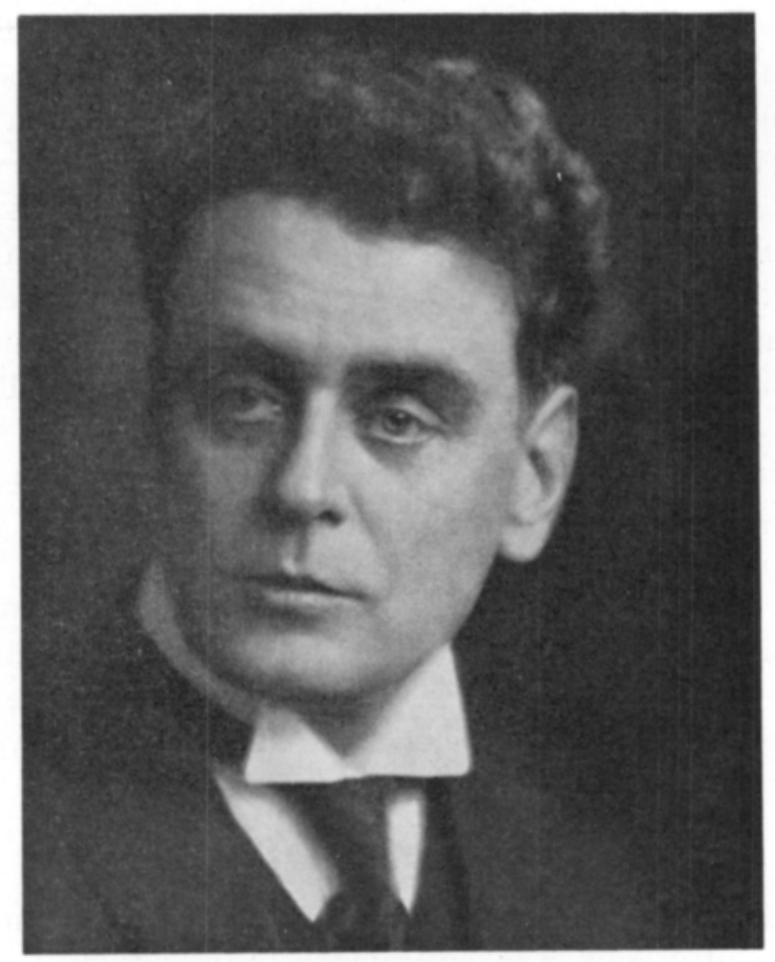

\title{
JONNI OTTO SAULI
}

* 3. 10. $1881-\dagger 16.8 .1957$

Nachruf, gehalten von Professor OtTo Valle auf der Sitzung der Finnischen Landwirtschaftswissenschaftlichen Gesellschaft am 21. Januar 1958.

Am 16. August vorigen Jahres starb in Helsinki im Alter von 75 Jahren der Professor für Pflanzenbau Jonni OTTo SaUli. Ein langes und reichhaltiges Tagewerk hatte er est als Pflanzenzüchter in der Pflanzenzuchtanstalt Tammisto und später als Professor für Pflanzenbau an der Universität Helsinki vollbracht. Im Jahre 1949 trat er als Emeritus in den Ruhestand.

J. O. Sauli wurde am 3.10.1881 in Hämeenlinna geboren. Sein Vater war der Fabrikant und Geschäftsmann Carl Otto Saxelin und seine Mutter Saida Maria (geb. Blåfield) Vertreterin eines alten finnischen Kulturgeschlechtes. Seine Kinderund zum Teil seine Jugendjahre verbrachte Sauli in seiner Geburtsstadt Hämeenlinna, die Reifeprüfung bestand er 1903 jedoch in Helsinki. Er war das älteste von fünf Kindern. Brüder von ihm waren der später als Bildhauer berühmt gewordene und jung verstorbene Into Saxelin und der als Schriftsteller bekannte Jalmari Sauli.

Jonni Sauli begann nach bestandener Reifeprüfung in der jungen Landwirtschaftlichen Sektion der Philosophischen Fakultät der Universität Helsinki Land- 
wirtschaftswissenschaften zu studieren. Als junger Student praktizierte Sauli einen Sommer in Hattula an der Landwirtschafsbotanischen Versuchsstation unter der Leitung von Mikael von Essen. Im Jahre 1906 stellte ihm sein Lehrer Professor Gösta Grotenfelt ein Landgerstenmaterial zur Verfügung, das teils von dem Botanikprofessor Fredrik Elfving schon im Jahre 1897 eingebracht, teils 1901 in verschiedenen Gegenden Finnlands gesammelt worden war. Die Variationen und Formen dieses finnischen Landgerstenmaterials erforschte Sauli in einer Spezialuntersuchung. Das Staatsexamen bestand er 1907, und gleich nach Beendigung seiner Studien widmete er sich seiner eigentlichen Lebensaufgabe im Dienste der Pflanzenerzeugung Finnlands.

Die erste Wirkungsstätte Saulis war der Finnische Saatzuchtverin (Suomen Kylvösiemenyhdistys), gegründet 1904, der ihm im Jahre 1907 in der Nähe von Helsinki, in Korso, einen Versuchsbetrieb für seine Tätigkeit zur Verfügung stellte. Sauli arbeite als Assistent des Saatzuchtvereins in den Jahren 1908-13. In diesen Jahren erhielt er eine ausgezeichnete Ausbildung für künftige anspruchsvollere Aufgaben. Der Saatzuchtverein betrieb umfassende Massnahmen zur Förderung des Samenbaues. Auf den Versuchsfeldern von Korso wurde ein umfangreichs Material verschiedener Pflanzenarten aus verschiedenen Gegenden Finnlands gesammelt, und in Feldversuchen wurden die Anbaueigenschaften des beschafften Materials herausgestellt. Da der Finnische Saatzuchtverein eine ähnliche Zuchtstätte wie die des Schwedischen Saatzuchtvereins, die unter dem Namen Svalöv bereits zu Ruf gelangt war, zu gründen beabsichtigte, leitete man auch in Korso Pflanzenzüchtungsarbeiten ein. Im Winter machte die Samenkontrolle einen bedeutenden Teil seiner Arbeit aus. Im Jahre 1911 machte Sauli sich auf einer dreimonatigen Studienreise in Mitteleuropa (Deutschland, Schweiz und Österreich) mit Samenkontrolle und Samenbau vertraut.

Die Tätigkeit des Finnischen Saatzuchtvereins stand schon infolge der bescheidenen staatlichen Zuwendungen wirtschaftlich auf schwachem Boden. Seinen Leistungen brachte man kein Vertrauen entgegen. Daher empfand die Zentralgenossenschaft Hankkija, mit der im J. 1911 eine Samenabteilung gegründet worden war — ihr erster Direktor war Väinö Suuronen - das Bedürfnis, eine eigene Versuchsstation zu gründen. Diesen Gedanken betrieb und verwirklichte beneb Suuronen der Vorsitzende des Verwaltungsrates von Hankkija, Senator A. Osw. Kairamo. Hankkija beschloss, im J. 1913 eine eigene Pflanzenzüchtungs- und Versuchsstation zu gründen, und für sie wurde ein umfassendes Arbeitsprogramm restgelegt. Mit den Aufgaben des Leiters wurde Magister Jonni Sauli betraut, der schon fünf Jahre in Korso mit entsprechender Versuchsarbeit beauftragt gewesen war. Da noch nicht sogleich eine eigene Versuchsstation zur Verfügung stand, wurde mit der Arbeit im Sommer 1913 auf dem Landgut Senator Kairamos in Pekola bei Hattula in der Nähe von Hämeenlinna begonnen. Als Saulis Assistent wurde» Konsulent Vilho Auramo angestellt.

Die Versuchsstation hatte anfangs auch viele andere Aufgaben ausser der Pflanzensucht: Versuchsbau des von Hankkija verkauften Handelssaatgutes, das Einrichten von vergleichenden Versuchen und Saatzuchtversuchen, Reinkultur des Landsortenmaterials und Samenkontrolltätigkeit. Saulis Verdienst war es, dass 
man bald der Arbeit der Versuchsstation Vertrauen entgegenzubringen begann, so dass Hankkija zu nunmehr grösseren Aufwendungen bereit war, um ein eigenes Vetsuchsgut zu schaffen und die Arbeit zu erweitern.

Drei Vegetationsperioden dauerte die Versuchs- und Forschungsarbeit in Pekola an, und es wurde ein umfassendes Material als Grundlage für die Züchtung landwirtschaftlicher Nutzpflanzen gesammelt. Ein eigenes Versuchsgut wurde im Jahre 1915 in der Landgemeinde Helsinki erworben. Im folgenden Jahre erfolgte die Ubbersiedlung nach Tammisto, wo die für den Pflanzenbau Finnlands bedeutsam gewordene Pflanzenzüchtungsanstalt Tammisto seitdem schon über 40 Jahre gearbeitet hat. Auf Tammisto setzt Sauli ungeachtet der durch den ersten Weltkrieg verursachten Schwierigkeiten sein erfolgreiches Wirken auf dem Gebiet der Pflanzenzüchtung fort. Sauli hat denn auch als Bahnbrecher der finnischen Pflanzenzüchtungsarbeit seinen Namen unauschlöslich in die Geschichte der Pflanzenzucht Finnlands eingezeichnet. Nachdem Finnland selbständig geworden war, erweiterte sich die Pflanzenzüchtungs- und Versuchstätigkeit beträchtlich.

Sauli bestand seine Doktorsdisputation im Jahre 1922 mit seiner Untersuchung "Erblichkeitsstudien an der Mustiala-Kohlrübe" (Abh. der Agrikulturwissensch. Gesellschaft Finnland 11). Mit diesem Forschungsstoff hatte er sich schon lange befasst, denn in Hankkijas eigener - im allgemeinen alle fünf Jahre mit einem Heft erscheinenden - Schriftenfolge "Siemenjulkaisu» war schon im Jahre 1914 ein Aufsatz über dieses Thema veröffentlich worden. Lic. phil. wurde Sauli 1924, und zum Dozenten der Pflanzenbaulehre an der Universität Helsinki wurde er 1928 ernannt.

Nachdem die Professur für Pflanzenbau an der Universität Helsinki 1929 schon zum dritten Mal ausgeschrieben worden war, hatte Sauli Intresse für diese neue Aufgabe bewarb sich um den Lehrstuhl. Vom 1. September 1929 an übernahm Sauli die Professur für Pflanzenbau i.V. Von da an hatte Sauli dieses Lehramt 20 Jahre inne. Zum ordentlichen Professor für Pflanzenbau wurde Sauli am 2. 2. 1934 ernannt. In den frühen 1930er Jahren wirkte Sauli in fünf Sommern (1930-34) jedoch noch als Pflanzenzüchter auf Tammisto, so dass er sich auch in dieser Aufgabe 20 Jahre betätigte.

Damit ist der äussere Rahmen umrissen worden, in dem Saulis Arbeit als Pflanzenzüchter und in der Versuchstätigkeit vor sich ging. Seine Arbeit als Pflanzenzüchter begann echt bald bedeutsame Ergebnisse zu bringen. Die meisten der von ihm entwickelten Zuchtsorten fanden Eingang in die Praxis in den ersten Jahrzehnten der Selbständigkeit Finnlands, in einer Zeit, als in der sich intensivierenden Pflanzenerzeugung Finnlands des Landsortenmaterial namentlich in den mittleren und nördlichen Teilen des Landes schnell durch veredelte Sorten zu ersetzen war Einige dieser von Sauli entwickelten Zuchtsorten von Tammisto haben auch heute noch nicht ihre Bedeutung verloren.

Als erstes Erzeugnis der Pflanzenzüchtungsarbeit von Hankkija gelangte im Jahre 1919 der von Sauli entwickelte Ruskea kevätvehnä (Brauner Sommerweizen) auf den Markt. Diese Weizenzuchtsorte war von recht grosser Bedeutung in den 1920 er Jahren auch und später noch, als sich in den 1930er Jahren der Sommerweizenbau Finnlands stark zu erweitern begann. Erst Svalöfs Sommerweizen Diamant vermochte sie zu verdrängen. 
In den 1920er Jahren kamen mehrere von Sauli entwickelte zwei- und vielzeilige Gerstensorten auf den Markt [Piikkiö (1922), Uurainen (1922) sowie Halikko I und II (1923, 1925) waren zweizeilig; 4- oder 6-zeilig dagegen waren Lappi I und II (1924, 1930), Olli (1927) und Perttu (1929)]. Alle diese Gersten waren Linienzuchten aus finnischen Landgersten. Am bedeutsamsten unter ihnen ist offenbar die zu den frühesten Gerstensorten der Erde gehörende Olli; denn obgleich sie später in Finnland selbst in erster Linie durch Tammi ersetzt worden ist, hat sie in Kanada, besonders in der Provinz Alberta, eine sehr bemerkenswerte Anbauweite erlangt. Olli ist dort die früheste Gerstensorte und wird auch als Braugerste angebaut. Die obengenannten zweizeiligen Gerstensorten von Tammisto wurden später durch die für Finnland als Braugerste genehmigte dänische Binder ersetzt, und als Konkurrent der vielzeiligen Gersten von Tammisto trat Svalöfs Vega auf. — Die TammiGerste $(O l l i \times A s p l u n d)$, die 1938 in den Handel gelangte, nachdem Sauli schon seine Arbeit auf Tammisto beendet hatte, ist eigentlich auch eine seiner Erungenschaften. Diese standfeste frühe Sorte war 1955 in Finnland die am meisten angebaute Gerstensorte.

Am bedeutendsten gestalte sich die züchterische Arbeit Saulis auf dem Gebiete der Haferzüchtung. Alle Hafersorten von Tammisto sind aus Kreuzungen entstanden. Sauli legte den Grund zu der namhaften Haferzüchtungsarbeit von Tammisto. In den 1920er Jahren kamen 7 Hafersorten von Tammisto in den Hande, die alle als einen Elternteil die Linie 091 eines aus der Gegend von Kuopio herrührenden Landhafers hatten. Unter diese Sorten waren in den 1020er und 1930er Jahren sehr bekannt die Schwarzhafer Osmo I und II (1921 und 1922), der Weisshafer Esa (1922), der Gelbhafer Kytö (1925) und der Schwarzhafer Pelso (1925). Kytö fand als standfest, ergiebig und früh reifend auch im Ausland Beachtung.

Von den Winterweizensorten von Tammisto, an deren Entwicklung Sauli ebenfalls sehr interessiert gewesen ist, kamen schon in den 1920er Jahren viele [Rusopää (1920), Veredelter Villa-Weizen (1921) und Sukkula I und II (1922 und 1928)] auf den Markt. Saulis bedeutendste Leistung auf dem Gebiet der Züchtung von Winterweizen ist der 1933 auf den Markt gelangte Varma, der auch heute noch - nach 25 Jahren - der meistbebaute Winterweizen in Finnland ist, obgleich danach auch zahlreiche neue Sorten auf den finnischen Saatgutmarkt gekommen sind.

Auch auf dem Gebiet der Erbsenzucht waren die Erfolge Saulis bedeutend. Die im Jahre 1923 in den Handel gekommene Kellervä war eine sehr ergiebige und schmackhafte Zuchtsorte, wurde aber wegen ihrer bunten, gelbgrünen Farbe durch andere Sorten ersetzt. In den 1930er Jahren hörte die Erbsenveredlung in Tammisto auf, um die Arbeitsteilung mit der staatlichen Pflanzenzuchtsanstalt in Jokioinen einzurichten. Das alte Züchtungsmaterial von Tammisto wurde jedoch bis zu Ende erprobt, und aus diesem Material Saulis rührt die 1952 im Handel erschienene Kalle-Erbse her, deren Anteil im Jahre 195564 \% der gesamten Erbsenfläche Finnlands ausmachte.

Ohne des weiteren alle Leistungen Saulis als Pflanzenzücher aufzuzählen, sei noch die von ihm entwickelte'Kartoffelsorte Tammiston aikainen (Tammistos Frühe) angeführt, die 1930 in den Handel kam und in den 1930er und auch noch in den 1940- 
er Jahren die beliebste Frühkartoffel in Finnland war. Sie verlor ihre Bedeutung in erster Linie aus dem Grunde, weil sie nicht krebsfest ist.

Neben der oben beschriebenen überaus erfolgreichen Pflanzenzüchtungsarbeit fand Sauli auch Zeit zu eingehender Forschungsarbeit wie auch zur Weitergabe seiner umfassenden Erfahrungen an die Bauernkreise Finnlands. Sauli leistete eine unersetzliche Arbeit, indem er sog. Landsortenmaterial in erster Linie an Winterweizen, Gerste und Hafer sammelte und Eigenschaften wie auch Herkunft dieser alten Anbauformen erforschte. Im Jahre 1925 erschien in der Schriftenreihe "Siemenjulkaisu" Saulis Studie "Suomessa viljellyt maatiaissyysvehnät" (Die in Finnland angebauten Landsorten des Winterweizens). Auf Grund dieser Schrift kann geschlossen werden, dass im Verlauf der Zeiten Winterweizen sowohl aus dem Osten als auch aus dem Westen nach Finnland gekommen sind. Im Jahre 1927 veröffentlichte Sauli die Arbeit "Suomen maatiaisohrat ja niiden jalostusarvo" (Die finnischen Landgersten und ihr züchterischer Wert; deutsches Ref., Acta Agralia Fennica 16). Bei der Zusammenstellung dieser Untersuchung wertete Sauli das bereits oben erwähnte um die Jahrhundertwende gesammelte Landgerstenmaterial aus. Dazu brachte Sauli in den Jahren 1919 und 1920 ein umfassendes Landgerstenmaterial aus den verschiedenen Gegenden Finnland sein. Nach dieser Untersuchung ist die 6-zeilige Gerste wahrscheinlich die erste Gerstenform in Finnland gewesen; die 4-zeilige ist jünger, und die 2-zeilige hat sich in Finnland vielleicht erst im 18. Jahrhundert auszubreiten begonnen. Im Jahre 1937 veröffentlichte Sauli seine Untersuchung "Suomen maatiaiskaurat" (Die finnischen Landhafer; deutsches Ref., Acta Agralia Fennica 34, 1). Mit seinen Untersuchungen finnischer Landhafer hatte Sauli schon im J. 1909 an der Versuchsstation des Finnischen Saatzuchtvereins begonnen, aber noch in den Jahren 1919 und 1920 sammelte er ein umfassendes Hafermaterial für seine Forschungen. Er stellte fest, dass die finnischen Landhafer dunkelkörnig waren, wie auch in Schweden. Dagegen sind alle aus dem Osten gekommen Hafer hellkörnig gewesen.

Diese Untersuchungen Saulis über die alten Formen der finnischen selbstbefruchtenden Getreidepflanzen Finnlands sind meines Erachtens die wertvollsten seiner wissenschaftlichen Leistungen. Es ist nur zu beklagen, dass sie alle auf finnisch erschienen sind, so dass sie im allgemeinen ausländischen Forschern entgangen sind. Sauli bemühte sich um das Einbringen des Landsortenmaterials der finnischen Getreidepflanzen zum letztmöglichen Zeitpunkt, denn schon in dẹn 1920er Jahren begannen sich in erster Linie die Zuchtsorten von Tammisto schnell in die verschiedenen Gegenden besonders Nord- und Mittel-Finnlands auszubreiten. Ein für die Pflanzenbaugeschichte Finnlands sehr aufschlussreiches Landsortenmaterial behandelte Sauli im J. 1934 in seiner Antrittsrede "Katsaus viljalajiemme kehitysvaiheisiin Suomessa (Ưber die Entwicklungsphasen der Getreidearten in Finnland; deutsches Ref., Acta Agralia Fennica 31, 8).

In einem bedeutenden finnischen land- und forstwirtschaftlichen Handbuch, „Maa ja metsä», und zwar in dem 1929 erschienenen Teil über den Pflanzenbau, stellte Sauli eine Fülle von Kenntnissen und Erfahrungen aus zwanzigjähriger Tätigkeit in Pflanzenzucht und Versuchswesen zusammen. Auch in vielen Leitfäden für engere Gebiete wertete Sauli seine umfassenden Kenntnisse vom tin- 
nischen Pflanzenbau aus. In Tammistos Berichten "Siemenjulkaisu" findet sich reichliches Material aus Saulis schriftlichem Schaffen.

Die Sachverständigkeit Saulis als Experte im Versuchswesen und als Landwirtschaftsforscher hat man auf mancherlei Weise ausgewertet. Als Finnland selbständig wurde und man die Samenkontrolltätigkeit erst einmal vorläufig einrichtete, wurde er 1918 zum interimistischen Direktor der Samenkontrollanstalt ernannt, und als die Staatliche Samenkontrollanstalt 1919 ihre Tätigkeit aufnahm, wurde er von Anfang an Mitglied ihres Vorstandes, als dessen Vorsitzender er von 1922 - 33 wirkte. Als Mitglied des Zentralausschusses des Landwirtschaftlichen Versuchswesens war Sauli von 1935-49 tätig, als Vorsitzender des vom Zentralverband der Landwirtschaftlichen Gesellschaften gegründeten Ausschusses für Standardisierung der Getreide-, Erbsen- und Kartoffelsorten von 1930-49. An der Tätigkeit der Landwirtschaftswissenschaftlichen Gesellschaft in Finnland beteiligte sich Sauli mit Begeisterung, und er wirkte 1929 als ihr Zweiter Vorsitzender, 1930 als ihre Erster Vorsitzender und 1931-35 als ihr Schriftführer. Ausserdem war er Mitglied zahlreicher anderer wissenschaftlicher Gesellschaften. Im Jahre 1936 wurde Sauli zum Ehrenmitglied des Schwedischen Saatzuchtvereins (Svalöv) und 1952 zum Ehrenmitglied der Landwirtschaftswissenschaftlichen Gesellschaft in Finnland ernannt.

Sauli war als Persönlichkeit rücksichtsvoll und angenehm. Als bescheidener Charakter, seiner Arbeit getreu, still und emsig, fühlte Sauli sich am wohlsten unter seinen lieben Pflanzen. Er hatte als Pflanzenzüchter eine phänomenale Fähigkeit und Geschicklichkeit, selbst bei einem umfassenden Züchtungsmaterial eine erfolgreiche Wahl zu treffen. Winters untersuchte er im Laboratorium sein sommers auf den Versuchsfeldern gewachsenes Material, und sommers machte er mit scharfem Auge seine Beobachtungen auf den weiten Versuchsfeldern von Tammisto.

Sauli konnte etwa zwei Jahrzehnte als Lehrer an der Universität Helsinki tätig sein; doch hat er zweifellos besonders mit seiner Pflanzenzüchtungsarbeit eine sehr tiefe Furchte in die Entwicklung der Landwirtschaft Finnlands eingezeichnet. Der Pflanzenerzeugung des selbständigen Finnland hat Sauli in einer wichtigen Entwicklungszeit besonders in den 1920er und 1930er Jahren grosse Dienste geleistet. In Anerkennung dieser seiner grossen Errungenschaften hat Suomen Kulttuurirahasto (Finnischer Kultur-Fond) ihm 1956 einen Ehrenpreis bewilligt.

\section{JONNI OTTO SAULI}

3. 10. $1881-16.8 .1957$

Helsingin Yliopiston täysinpalvellut kasvinviljelytieteen professori J. O. Sauli kuoli Helsingissä 16/8 1957. Hän työskenteli Hankkijan kasvinjalostuslaitoksen johtajana lähes pari vuosikymmentä ja vuodesta 1929 alkaen aluksi väliaikaisena ja vuodesta 1934 lähtien vakinaisena kasvinviljelytieteen professorina eläkkeelle siirtymiseensä saakka v. 1949.

Saulin elämäntyőstä muodostui merkittävimmäksi hänen toimintansa kasvinjalostuksemme uranuurtajana. Useita kymmeniä Saulin kehittämiä jalosteita joutui 1920- ja 1930-luvuilla siemenmarkkinoille. Eräăt niistä eivät ole vieläkään menettäneet merkitystään.

Saulin tieteellisestä tutkimustoiminnasta on mainittava erityisesti hänen työnsä suomalaisten maatiaissyysvehnien, maatiaisohrien ja maatiaiskaurojen muotojen ja alkuperän selvittämiseksi.

Suomenkielisen kuvauksen Saulin elämäntyöstă on kirjoittaja esittănyt Pellervossa (n:o 1, 1958). 